\title{
Complete Genome Sequence and Biological Characterization of Moroccan pepper virus (MPV) and Reclassification of Lettuce necrotic stunt virus as MPV
}

\author{
William M. Wintermantel and Laura L. Hladky
}

United States Department of Agriculture-Agricultural Research Service, 1636 East Alisal Street, Salinas, CA 93905. Accepted for publication 19 December 2012.

\begin{abstract}
Wintermantel, W. M., and Hladky, L. L. 2013. Complete genome sequence and biological characterization of Moroccan pepper virus (MPV) and reclassification of Lettuce necrotic stunt virus as MPV. Phytopathology 103:501-508.

Moroccan pepper virus (MPV) and Lettuce necrotic stunt virus (LNSV) have been steadily increasing in prevalence in central Asia and western North America, respectively, over the past decade. Recent sequence analysis of LNSV demonstrated a close relationship between the coat proteins of LNSV and MPV. To determine the full extent of the relationship between LNSV and MPV, the genomes of three MPV isolates

were sequenced and compared with that of LNSV. Sequence analysis demonstrated that genomic nucleotide sequences as well as virus-encoded proteins of the three MPV isolates and LNSV shared $97 \%$ or greater identity. A full-length clone of a California LNSV isolate was developed and virus derived from infectious transcripts was used to evaluate host plant reactions under controlled conditions. Symptoms of LNSV matched those described previously for MPV on most of a select series of host plants, although some differences were observed. Collectively, these molecular and biological results demonstrate that LNSV should be classified as MPV within the family Tombusviridae, genus Tombusvirus, and confirm the presence of MPV in North America.
\end{abstract}

A tombusvirus related to Tomato bushy stunt virus (TBSV) was first described from pepper in Morocco, and produced symptoms including leaf deformation, stunting, leaf and flower abscission, and lethal systemic necrosis upon mechanical inoculation to pepper plants (4). The virus was named Moroccan pepper virus (MPV) in 1981 (13), and has been recognized for many years as a partially characterized member of the genus Tombusvirus, family Tombusviridae. MPV was also isolated in southern Germany in the early 1980s from naturally infected tomato plants exhibiting mottle and malformation of upper leaves and shoot tips, as well as from pelargonium plants exhibiting leaf malformation and stunting, with infection confirmed through serological studies and host plant inoculations (19). More recently, several isolates of MPV have been identified from lisianthus (Eustoma grandiflorum) (2), tomato (Solanum lycopersicum), and roots of jimson weed (Datura stramonium) in Iran (15).

Another tombusvirus, Lettuce necrotic stunt virus (LNSV) was identified as a causative agent of lettuce dieback disease in California and Arizona in the 1990s $(12,14)$. Lettuce dieback is characterized by stunting and death of lettuce plants and often occurs in low-lying areas with poor drainage, in areas near rivers, on recently flooded land, and in areas where soil has been dredged from a river or ditch and spread onto adjacent fields $(12,14,22)$. Most non-crisphead lettuce lines are susceptible to

Corresponding author: W. M. Wintermantel;

E-mail address: bill.wintermantel@ars.usda.gov

* The $\boldsymbol{e}$-Xtra logo stands for "electronic extra" and indicates that the online version contains two supplemental figures. Figures 1 to 4 appear in color online. The online version contains three links to new GenBank accession numbers JX182425 (MPV isolate EM81), JX197070 (MPV isolate PG82), and JX197071 (MPV isolate PM75).

http://dx.doi.org/10.1094/PHYTO-07-12-0166-R

This article is in the public domain and not copyrightable. It may be freely reprinted with customary crediting of the source. The American Phytopathological Society, 2013. infection, and some fields cannot be planted with non-crisphead lettuce due to the severity of the problem. LNSV also affects glasshouse tomato production and has a substantial, partially characterized host range similar to that of several members of the genus Tombusvirus (14).

Recent sequencing of the genome of the L2 isolate of LNSV from lettuce (JN700748) demonstrated a high level of conservation between the LNSV coat protein and that of MPV (21), the only region of the MPV genome that had been sequenced to date. The coat protein amino acid sequence of the LNSV-L2 isolate shares 94 to $99 \%$ amino acid identity with the aligned coat proteins of six isolates of MPV available in GenBank (accession numbers AEW27316, ACA48477, ACA48478, ABZ05023, AAN28378, and ADZ45531). The high degree of sequence identity between the coat proteins of LNSV and MPV isolates suggests a very close relationship between the two viruses, because most distinct members of the Tombusvirus genus have widely divergent coat proteins. The recent emergence of MPV isolates in Central Asia in both crops and soil $(2,15)$ warrants a thorough evaluation of the MPV genome, including comparative host range analysis, to determine whether MPV and LNSV are, indeed, the same virus or closely related species, as well as development of an infectious clone for preservation and study.

\section{MATERIALS AND METHODS}

Virus isolates and sequence analysis. The type isolate of MPV (MPV-Type) was collected from pepper (Capsicum annuum) in the Rabat-Salé region of Morocco in 1975 (4), and was first introduced as MPV in 1981 (13). The isolate, which was propagated in Nicotiana benthamiana, was kindly provided by M. Russo and H. J. Vetten. Total RNA was extracted from dried $N$. benthamiana leaf tissue using the Qiagen RNeasy Plant Mini Kit (Qiagen, Valencia, CA) per the manufacturer's instructions. Two additional isolates from pelargonium (Pelargonium sp.) and eggplant (S. melongena) were generously provided as purified 
virus extracts by H. J. Vetten and R. Koenig for use in the sequence comparison studies. The pelargonium isolate (identified herein as MPV-PG82) was originally obtained in 1982 from diseased plants in Germany (19). The eggplant isolate (identified herein as MPV-EM81) was collected in 1981 from the Rabat-Salé region of Morocco, and was maintained in tomato (S. lycopersicum) by R. Koenig. The L2 isolate was originally isolated from infected lettuce plants near Salinas, CA (14) and was maintained in $N$. clevelandii and $N$. benthamiana. The LNSV-L2 isolate was recently sequenced (21) and used for comparison with the MPV sequences and for preparation of an infectious clone.

Polymerase chain reaction (PCR) primer sets previously designed for sequencing of the LNSV-L2 genome (21) were used to sequence the entire genomes of all three MPV isolates. Reverse transcription was performed using the LNSV-L2 primer 4772R $\left(5^{\prime}\right.$ ATATCCCGGGGGGCTGCATTTCTGCAATG $\left.3^{\prime}\right)$ or 2723R (5' CAAATGCTTTCTGGAGGCCACGTT 3') and Superscript III reverse transcriptase (Life Technologies, Carlsbad, CA) per the manufacturer's protocols. The resulting cDNA was PCR amplified in short $(<1,000 \mathrm{bp})$ sections using overlapping LNSV-L2 primer sets and Platinum Pfx DNA polymerase (Life Technologies) to obtain full coverage of the MPV genomes. Standard manufacturer's protocols were used in all reactions. The PCR amplicons were then directly sequenced in both forward and reverse directions (TACGen, Richmond, CA). In addition, the cDNA of isolate MPV-EM81 was amplified in two overlapping sections using LNSV-L2 primers 1F (5' ATTCTAGAAGAA ATTCCCCAGGATTTCTCG $\left.3^{\prime}\right)$ with $2723 \mathrm{R}$ and 2229F $\left(5^{\prime}\right.$ TCCGCACTGCAATGAGCAAAGATG 3') with 4772R using Platinum Pfx DNA polymerase and manufacturer-recommended protocols. These reactions were facilitated by use of the PCRx Enhancer Solution included with the kit, annealing at $50^{\circ} \mathrm{C}$, and an increased extension cycle of $3 \mathrm{~min}$. Each section was cloned into a pGem-T Easy vector (Promega Corp., Madison, WI) after A-tailing with poly-A-polymerase (Life Technologies). Two separate clones of each MPV-EM81 section were fully and bidirectionally sequenced, aligned, and compared with the sequencing results performed directly on each PCR amplicon. The consensus sequences for all three MPV isolates were assembled and analyzed using AssembyLIGN (MacVector 7.2; Accelrys, San Diego, CA). Protein sequences were determined using Open Reading Frame (ORF) Finder (http://www.ncbi.nlm.nih.gov/ gorf/gorf.html). The nucleotide and protein sequence alignments among MPV isolates and other tombusviruses were performed using ClustalW2 (www.ebi.ac.uk/Tools/msa/clustalw2) and MacVector 7.2.

Preparation of a LNSV infectious clone. To prepare the fulllength infectious clone of LNSV-L2, total nucleic acid was extracted from infected $N$. clevelandii using the method of Dellaporta and colleagues (3). The virus was amplified in two overlapping fragments using the same primer sets as for the MPVEM81 clones described above. Primers $1 \mathrm{~F}$ and 4772R contain $X b a \mathrm{I}$ and $S m a \mathrm{I}$ sites, respectively, to facilitate development of the full-length clone. The two fragments were cloned separately into either the ZeroBlunt TOPO vector (Life Technologies) or A-tailed and cloned into the pGEM-T Easy vector. The two LNSV clones were ligated together into a pUC18 vector by cutting at the $\mathrm{XbaI}$ and SmaI sites and utilizing an interior common XhoI site contained in each section of the LNSV clone. The resulting fulllength LNSV insert was then removed from pUC18 and cloned into pBluescript II SK(+) between the SalI and SacI sites for utilization of the Bluescript T7 promoter. Capped RNA transcripts were synthesized using the mMessage mMachine T7 Ultra kit (Life Technologies) according to the manufacturer's recommendations, after linearizing with SmaI, and using the optional proteinase $\mathrm{K}$ treatment. The quality and approximate quantity of the transcript was assessed using $1 \mu \mathrm{l}$ of the product in a $1 \%$ agarose gel. The infectious RNA was verified by inoculation to seedlings of $N$. benthamiana, $N$. clevelandii, and Chenopodium quinoa (all at four-leaf stage) after dusting leaves with carborundum. Approximately $4 \mu \mathrm{g}$ of RNA (mixed 1:1 with sterile $0.5 \mathrm{M}$ sodium phosphate buffer, $\mathrm{pH} 7.2$ ) was gently rubbed onto leaves of each plant using a sterile ceramic pestle. Symptoms were observed on all plants within 5 days and infection was confirmed by reversetranscription (RT)-PCR and direct sequencing of virus RNA isolated from noninoculated leaves. An infectious clone, pTBSV100 (7), of TBSV-Cherry strain (TBSV-Ch) was obtained as a gift from $\mathrm{H}$. B. Scholthof for use in comparative host range analysis with the LNSV-L2 clone. RNA synthesis and transcript inoculation from this clone was conducted as described above for the clone of LNSV-L2.

Host range and comparative symptom analysis. Clone derived LNSV-L2 and TBSV-Ch RNA transcripts were mechanically inoculated to healthy young $N$. benthamiana plants to establish fresh infections of each virus. Use of clone-derived viral RNA to initiate infection was critical in order to minimize potential influence of defective interfering RNAs (diRNAs) on symptom development. Transcript-inoculated $N$. benthamiana plants began exhibiting symptoms of infection by each virus within 5 to 7 days. As soon as systemic symptoms began to appear, N. benthamiana leaves were harvested and frozen to preserve inoculum for use in host range and symptom evaluation. These frozen stocks of LNSV-L2- and TBSV-Ch-infected $N$. benthamiana derived from transcript inoculations were used to inoculate a series of host plants to determine differential symptom responses between the two viruses, and for comparison with previous studies differentiating MPV symptoms with those of TBSV $(4,19)$. Test plants inoculated with each virus included Capsicum annuum ('California Wonder' bell pepper), Chenopodium quinoa, $C$. murale, C. capitatum, Cucurbita pepo ('Black Beauty' zucchini squash), D. stramonium, Lactuca sativa ('Valmaine' lettuce), $N$. benthamiana, $N$. clevelandii, N. glutinosa, S. lycopersicum ('SRT' tomato), and Spinacea oleracea ('Giant Thick Leafed' spinach). Plants were maintained in a Conviron PGR15 growth chamber at $25^{\circ} \mathrm{C}$ with 16 -h day length. Light intensity at plant level was maintained at $220 \mu \mathrm{E} \mathrm{m}{ }^{-2} \mathrm{~s}^{-1}$. Healthy plants of each host plant were maintained without inoculation for comparison in each experiment. Symptoms were evaluated at 6 and 14 days following inoculation, with continued monitoring until 28 days after inoculation. Host range and symptomatology inoculation experiments with LNSV-L2 were repeated four times, twice in parallel with TBSV-Ch inoculations.

\section{RESULTS}

The genomes of all three MPV isolates (accession numbers JX197070, JX197071, and JX182425) are 4,772 nucleotides (nt) in length, and contain all of the major ORFs characteristic of viruses in the genus Tombusvirus (family Tombusviridae) (20). The $5^{\prime}$ end of the genome contains a 166-nt untranslated region (5' UTR). ORF1 (nucleotides 167 to 1057 ) encodes a 33-kDa protein. ORF2 (nucleotides 167 to 2623) encodes a 92-kDa protein and is expressed by read-through of an amber stop codon at nucleotides 1055 to 1057 , resulting from a type III regulatory signal immediately $3^{\prime}$ of the UAG (1), and identical to the signal sequence present in TBSV. The P92 protein includes the GDD motif characteristic of RNA-dependent RNA polymerases at amino acids 625 to 627 . Both the P33 and P92 proteins are required for viral replication based on homology to other tombusviruses (20). ORF3 (nucleotides 2645 to 3829), which encodes the $41-\mathrm{kDa}$ virus coat protein, shares $98 \%$ identity with LNSV (accession number JN700748) (21) but only 64\% identity with TBSV-Ch, its next closest genetic relative among fully sequenced members of the genus. The high degree of sequence identity between the coat proteins of LNSV-L2 and MPV isolates indicates a very close relationship between the two viruses, because most 
distinct members of the genus have widely divergent coat proteins. ORF4 (nucleotides 3854 to 4423) and ORF5 (nucleotides 3886 to 4404 ) encode the P22 and P19 proteins, respectively.

Direct comparison of genomic RNA and virus proteins between MPV and LNSV demonstrate an exceptionally high level of conservation between these viruses. All three isolates of MPV and the LNSV-L2 isolate are exactly 4,772 nt in length. Genomic RNA of LNSV-L2 (JN700748) is 97\% identical to that of each of the three MPV isolates. Similarly, the MPV-Type isolate (JX197071) shares 98\% genomic identity with MPV-PG82 (JX197070) and MPV-EM81 (JX182425). MPV-PG82 and MPVEM81 share 99\% identity. The 5' UTRs of both LNSV-L2 and MPV-EM81 are identical, with only 3 nt differences between LNSV and MPV-Type. The $110 \mathrm{nt}$ at the $3^{\prime}$ end of the genome beyond ORF6 are also identical between LNSV-L2 and MPVType. The P33 replicase proteins of LNSV-L2 and MPV-Type are identical, with MPV-PG82 and MPV-EM81 differing by 1 and 2\% respectively (Table 1). P92 and CP share 99 and $98 \%$ identity among all four isolates, respectively. The P19 and P22 proteins share 97 to $98 \%$ identity among the four isolates, with LNSV differing from MPV-Type by $3 \%$ and MPV-PG82 and MPVEM81 differing by $2 \%$ for both proteins (Table 1).

Analysis of protein identities and similarities between MPV and other tombusviruses is virtually identical to that between LNSV-L2 (JN700748) and other tombusviruses (Table 1) (21). Overall, both viruses are most closely related to TBSV but also share close relationships with Artichoke mottled crinkle virus (AMCV) and Grapevine Algerian latent virus within the replication proteins, and with Carnation Italian ringspot virus and AMCV within P19 and P22.

Host range and symptom analysis using infectious transcripts of LNSV-L2 and TBSV-Ch. Inoculation of capped transcripts derived from the cloned LNSV-L2 isolate resulted in development of necrotic lesions on Chenopodium quinoa and systemic infection of $N$. clevelandii and $N$. benthamiana within 5 days. Aside from LNSV, TBSV is the closest genetic relative of MPV. In order to compare the host ranges of MPV and LNSV, replicated tests were conducted on a select set of host plants to illustrate similarities and differences among tombusviruses. Because it was not possible to propagate MPV due to permit restrictions, differences in host range between LNSV-L2 and TBSV-Cherry were examined under standardized growth-chamber conditions, and results were compared with previous studies comparing symptoms of MPV and TBSV on many of the same hosts. Test plants were inoculated with sap of virus-infected $N$. benthamiana plants inoculated with clone-derived transcripts of either LNSV-L2 or the TBSV-Ch strain. Host plants were selected based on previous studies demonstrating differential reaction to infection between MPV and TBSV (5,21), and included Capsicum annuum (bell pepper), D. stramonium, S. oleracea (spinach), Chenopodium quinoa, C. murale, C. capitatum, Cucurbita pepo (zucchini squash), L. sativa (romaine lettuce), N. benthamiana, N. clevelandii, N. glutinosa, and Solanum lycopersicum (tomato). The number of lesions was monitored on the second and third true leaves of $N$. glutinosa, a known local lesion host, in order to estimate relative concentration of each virus in the $N$. benthamiana tissue used in test inoculations (Fig. 1). An average of 41.1 lesions per leaf (LNSV) and 41.2 lesions per leaf (TBSV) over two separate experiments suggested that inoculum levels were comparable between the two viruses. Results of standardized and replicated host plant inoculations are summarized in Table 2 but some specific host reactions warrant greater detail.

Two sizes of $C$. quinoa plants were inoculated: seedlings at the two-true-leaf stage and larger plants $\approx 15 \mathrm{~cm}$ in height. Seedlings inoculated with either LNSV or TBSV developed necrotic lesions and systemic infection, resulting in death of all plants by 8 days postinoculation (Supplemental Figure 1A and B). Larger C. quinoa plants inoculated with LNSV developed necrotic lesions, and localized chlorosis on inoculated leaves, with a limited number of plants exhibiting systemic infection, including leaf deformation. Larger $C$. quinoa plants inoculated with TBSV developed necrotic lesions on the inoculated leaf but, unlike LNSV, no local chlorosis was observed (Supplemental Figure 1C and D). Some stem necrosis was observed on larger plants inoculated with either virus (Table 2). These symptoms are similar to those described previously comparing symptom development in Chenopodium quinoa plants inoculated with TBSV and MPV $(4,19)$. Similar results were observed on $C$. murale, with necrotic local lesions on inoculated leaves with both TBSV and LNSV; however, LNSV also induced spreading chlorosis on inoculated leaves that was not observed with TBSV infection. Symptoms of the two viruses were indistinguishable on $C$. capitatum (Table 2).

D. stramonium plants inoculated with TBSV developed chlorotic lesions on the inoculated leaf (Fig. 2B); however, D. stramonium inoculated with LNSV developed necrotic lesions and occasional leaf curling in areas where lesions occurred (Fig. 2A). Plants inoculated with TBSV resulted in systemic infection indicated by star-shaped chlorotic spots, many of which developed necrotic centers that cracked as the leaf expanded (Fig. 2D), whereas LNSV did not infect D. stramonium systemically (Fig. 2C) (Table 2). Spinach (Spinacea oleracea) plants inoculated with LNSV at the four-true-leaf stage developed chlorotic lesions on inoculated leaves eventually developing into systemic chlorosis, leaf curling, vein clearing, and stunted growth (Fig. 3A). In contrast, S. oleracea plants inoculated with TBSV developed necrotic local lesions on the inoculated leaves, leading quickly to tissue collapse and plant death (Fig. 3B). LNSVinfected $S$. oleracea plants collapsed more slowly, with some plants lasting as long as 3 weeks after inoculation (Table 2). Symptoms of LNSV on $S$. oleracea were milder than those previously described for MPV or $\operatorname{LNSV}(4,14,19)$, although

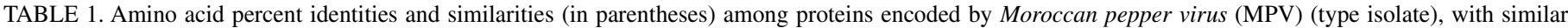
proteins from other tombusviruses ${ }^{\mathrm{a}}$

\begin{tabular}{|c|c|c|c|c|c|c|c|c|c|c|c|c|}
\hline Virus $^{\mathrm{b}}$ & MPV-PG82 & MPV-EM81 & LNSV-L2 & TBSV & $\mathrm{CNV}$ & AMCV & EMCV & GALV & PNSV & CymRSV & CBLV & CIRV \\
\hline P33 & $99(0)$ & $98(0)$ & $100(0)$ & $96(2)$ & $89(5)$ & $93(3)$ & $82(9)$ & $96(3)$ & $56(10)$ & $86(7)$ & $60(14)$ & $54(11)$ \\
\hline P92 & $99(0)$ & $99(0)$ & $99(0)$ & $94(2)$ & $93(2)$ & $94(3)$ & $89(6)$ & $94(3)$ & $79(5)$ & $90(4)$ & $76(9)$ & $78(5)$ \\
\hline $\mathrm{CP}$ & $98(1)$ & 98 (1) & $98(0)$ & 64 (13) & 34 (17) & $61(16)$ & $62(15)$ & 46 (17) & $39(17)$ & $42(15)$ & $35(20)$ & $56(14)$ \\
\hline $\mathrm{P} 22$ & $98(1)$ & $98(1)$ & 97 (1) & $97(1)$ & $89(6)$ & $96(1)$ & $88(6)$ & $88(7)$ & $88(8)$ & $84(8)$ & $81(10)$ & 97 (1) \\
\hline P19 & $98(0)$ & $98(0)$ & $97(0)$ & $90(4)$ & $71(9)$ & $88(4)$ & $73(9)$ & $73(8)$ & 72 (11) & $72(8)$ & $59(12)$ & $88(3)$ \\
\hline ORF6 $^{c}$ & 98 & 98 & 96 & 69 & 85 & 77 & 50 & 82 & NA & 83 & NA & 80 \\
\hline
\end{tabular}

a Homologies with $<25 \%$ identity over the length of the protein are not considered related; NA = not available.

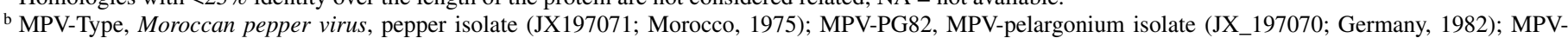
EM81, MPV-eggplant isolate (JX_182425; Morocco, 1981); LNSV-L2, Lettuce necrotic stunt virus, lettuce isolate (JN_700748; California, 1997; TBSV, Tomato bushy stunt virus (NC_001554); CNV, Cucumber necrosis virus (NC_001469); AMCV, Artichoke mottled crinkle virus (NC_001339); EMCV, Eggplant mottled crinkle virus (NC_004723); GALV, Grapevine Algerian latent virus (NC_011535); PNSV, Pelargonium necrotic spot virus (NC_005285); CymRSV, Cymbidium ringspot virus (NC_003532); CBLV, Cucumber Bulgarian virus (NC_004725); CIRV, Carnation Italian ringspot virus (NC_003500).

c Open reading frame (ORF)6 comparison is between nucleotide sequences. 
symptoms were highly consistent across all four replications of the experiment, and previous studies provided limited detail.

Pepper (Capsicum annuum) plants inoculated at the two-trueleaf stage developed chlorotic symptoms with both viruses within a few days. Plants inoculated with LNSV developed light green

A

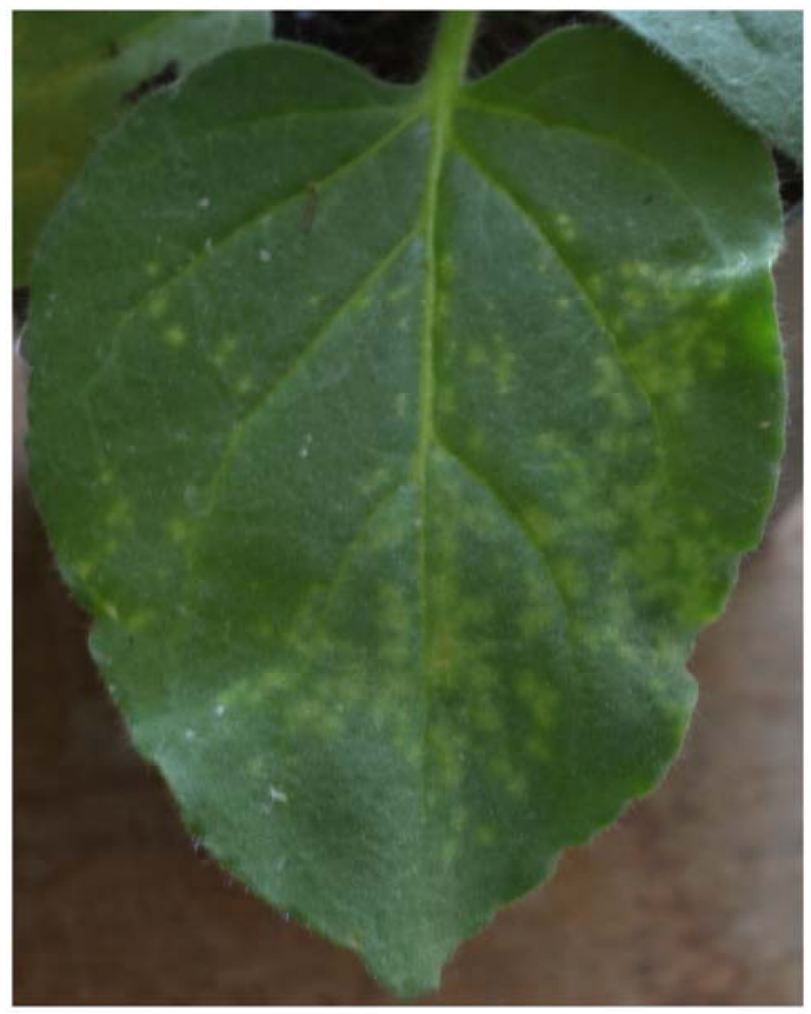

mosaic symptoms, eventually progressing into leaf deformation and upward curling (Fig. 4A), whereas plants inoculated with TBSV developed a bleached mosaic pattern but failed to exhibit the leaf curl symptoms (Fig. 4B). Comparative inoculations of pepper with LNSV and TBSV were repeated three times with
B

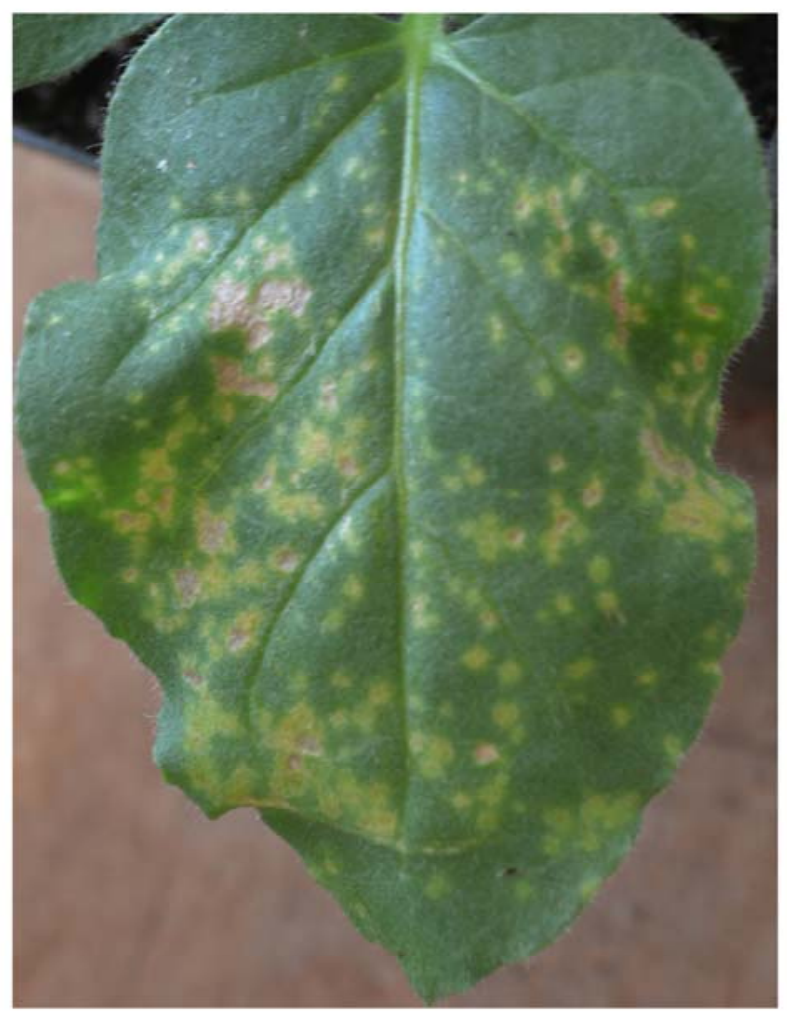

MPV (LNSV)-L2

\section{TBSV-Cherry}

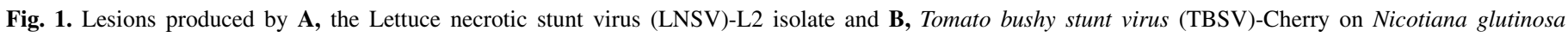
(14 days postinoculation).

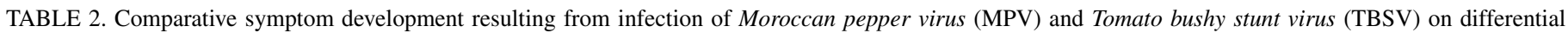
host plants ${ }^{\mathrm{a}}$

\begin{tabular}{|c|c|c|c|c|}
\hline \multirow[b]{2}{*}{ Host plant } & \multicolumn{2}{|c|}{ MPV (LNSV)-L2 } & \multicolumn{2}{|c|}{ TBSV-Ch } \\
\hline & Inoculated leaf & Systemic & Inoculated leaf & Systemic \\
\hline Spinacea oleracea & CLL & VC, LC, M, S, D & NLL, LTC & LTC, S, D \\
\hline Datura stramonium & NLL, LC & None & CLL, NS & $\mathrm{CS}^{\mathrm{b}}$ \\
\hline Chenopodium quinoa (small) & NLL, LY & S, LTC, D & NLL & S, LTC, D \\
\hline C. quinoa (large) & NLL, LY & $\mathrm{CS}, \mathrm{LC}, \mathrm{NS}, \mathrm{StN}$ & NLL, LTC & $\mathrm{CS}, \mathrm{LC}, \mathrm{NS}, \mathrm{StN}$ \\
\hline C. murale & NLL, LY & $\mathrm{S}, \mathrm{LD}, \mathrm{LC}, \mathrm{NS}^{\mathrm{b}}, \mathrm{D}$ & NLL & $\mathrm{S}, \mathrm{CS}, \mathrm{NS}, \mathrm{D}$ \\
\hline C. capitatum & NLL, LTC & LC, LTC, D & NLL, LTC & LC, LTC, D \\
\hline Solanum lycopersicum & CLL, LD & None & CLL, LD & None \\
\hline Lactuca sativa & CLL, M & None & NLL, LTC & None \\
\hline Nicotiana glutinosa & CLL, NLL & None & CLL, NLL & None \\
\hline N. clevelandii & CLL, LY, LTC & $\mathrm{M}, \mathrm{LD}, \mathrm{S}, \mathrm{D}$ & CLL, LY, LTC & $\mathrm{M}, \mathrm{LD}, \mathrm{S}, \mathrm{D}$ \\
\hline N. benthamiana & CLL, NLL, LTC & $\mathrm{M}, \mathrm{S}, \mathrm{LC}, \mathrm{D}$ & CLL, NLL, LTC & $\mathrm{M}, \mathrm{S}, \mathrm{LC}, \mathrm{D}$ \\
\hline Cucurbita реро & NLL, LC, LTC & None & NLL, LC, LTC & None \\
\hline Capsicum annuиm & $\mathrm{C}^{\mathrm{b}}$ & $\mathrm{C}, \mathrm{S}, \mathrm{M}^{\mathrm{b}}, \mathrm{LD}, \mathrm{LC}$ & $\mathrm{C}$ & $\mathrm{C}, \mathrm{S}, \mathrm{M}^{\mathrm{b}}, \mathrm{LD}$ \\
\hline
\end{tabular}

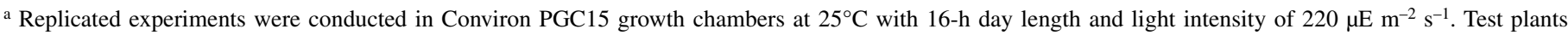
were inoculated mechanically with sap from 2-week-old $N$. benthamiana tissue infected systemically with either a single local lesion isolate of TBSV cherry strain (TBSV-Ch) or with transcripts derived from the cloned MPV (Lettuce necrotic stunt virus [LNSV])-L2 isolate. Plants were evaluated at 6, 14, and 21 days postinoculation. Symptoms: NLL, necrotic local lesions; CLL, chlorotic local lesions; LD, leaf distortion; LC, leaf curling; LY, localized yellowing around lesions; LTC, localized tissue collapse; M, mosaic; C, chlorosis; S, stunting; CS: chlorotic spots; NS, necrotic spots; VC, vein clearing; StN, stem necrosis; D, plant death.

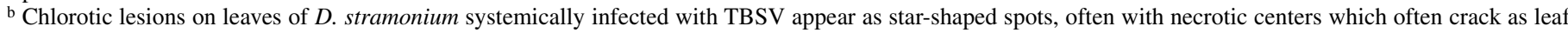
expands. Necrotic spots on leaves of Chenopodium murale systemically infected with MPV (LNSV)-L2 often have a necrotic halo or ringspot appearance. Chlorosis and mosaic symptoms on Capsicum annuum are lighter green with LNSV and appear as a foliar bleaching symptom with TBSV. 
highly consistent symptom development. Although previous reports indicated that MPV could also induce lethal systemic necrosis on pepper, this was not observed in these experiments.

Tomato (Solanum lycopersicum) plants inoculated at the six true-leaf stage produced similar symptoms for both viruses, resulting in leaf distortion and development of 3- to 4-mm chlorotic lesions on the inoculated leaf in most plants. Although both viruses induced development of a small number of necrotic lesions as well, this was more common for TBSV than LNSV. Neither virus infected tomato systemically in these experiments. Symptoms of both viruses on $N$. benthamiana, $N$. clevelandii, and $N$. glutinosa were very similar, as expected. When inoculated at the four-true-leaf stage with either virus, $N$. benthamiana plants developed systemic infection within 2 days, characterized by leaf curling and puckering. Systemic chlorosis developed on $N$. clevelandii. Collapse of $N$. benthamiana and $N$. clevelandii infected with either LNSV or TBSV usually occurred within 7 to 10 days. $N$. glutinosa developed necrotic local lesions when infected with either virus and did not infect plants systemically.

Systemic infection of lettuce by either LNSV or TBSV requires infection through roots under specific environmental conditions that were not applied during these experiments. Interestingly, when plants were maintained in the growth chamber with a $16-\mathrm{h}$ day length and $220 \mu \mathrm{E} \mathrm{m}^{-2} \mathrm{~s}^{-1}$ light intensity, LNSV-L2 produced extensive mosaic patterns on the inoculated leaves of some plants and chlorotic lesions on others, whereas TBSV-Ch strain induced necrotic lesions on inoculated leaves, often with lesions coalescing into larger areas of necrosis (Supplemental Figure 2). Previous studies in lettuce have demonstrated extreme variability in symptom development on this host depending on environmental conditions for both viruses (W. M. Wintermantel and I. Simko, unpublished data).

\section{DISCUSSION}

All genetic and host-range comparisons demonstrate a high degree of nucleotide and protein conservation between LNSV-L2 and the three MPV isolates. This is highlighted most clearly by the near identity between the coat protein of LNSV and that of each of the MPV isolates. The sequence of the coat protein is highly variable among Tombusvirus spp. and, therefore, is widely used to identify and differentiate tombusviruses from one another. The high level of amino acid sequence conservation within this protein between LNSV and MPV identified in our previous study
A

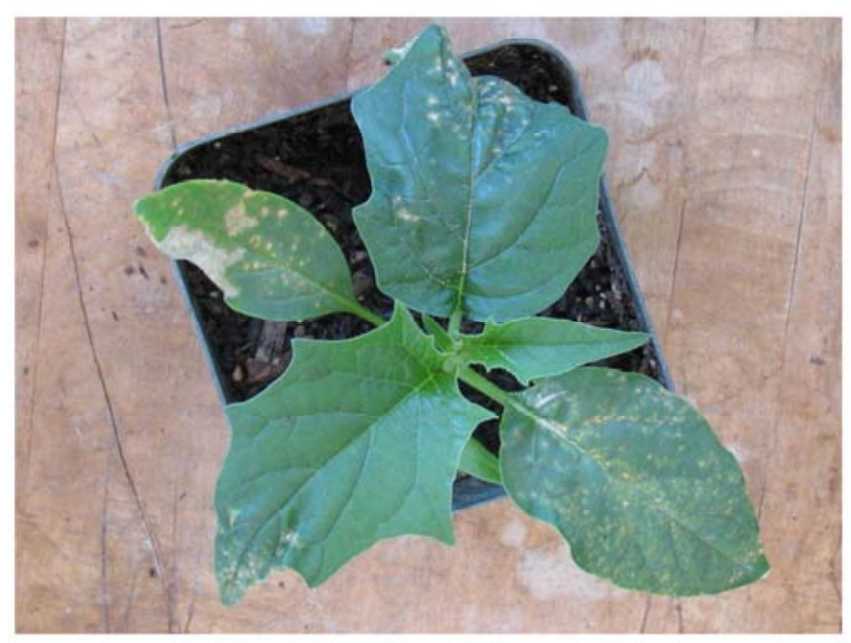

C

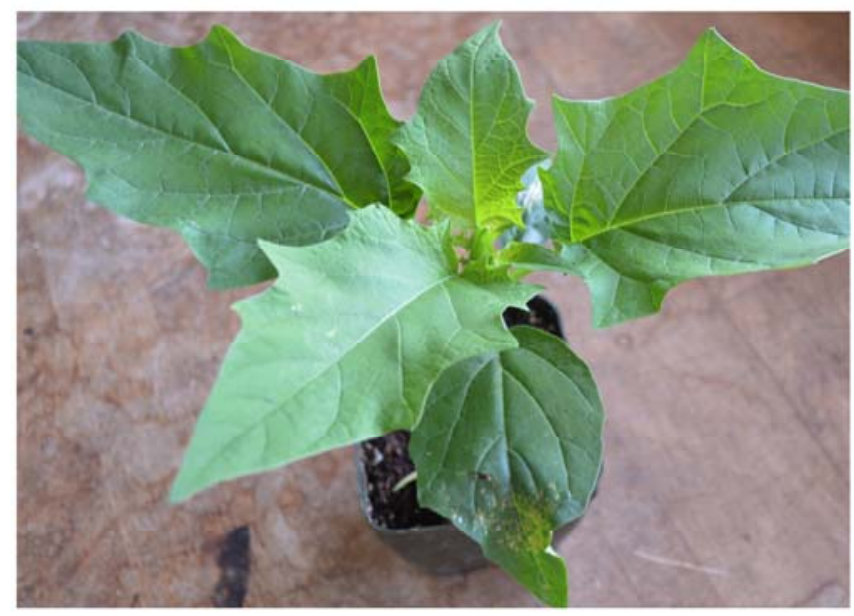

MPV-(LNSV)-L2
B

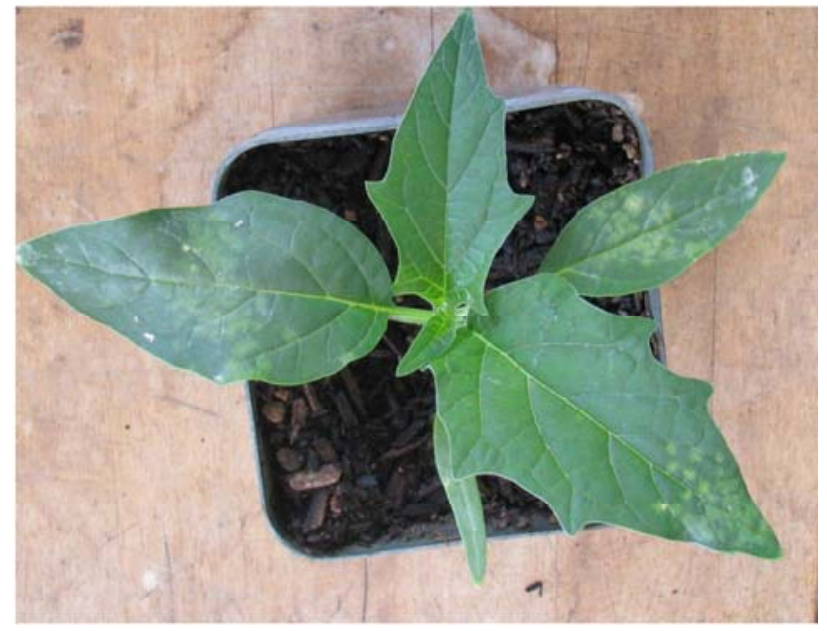

D

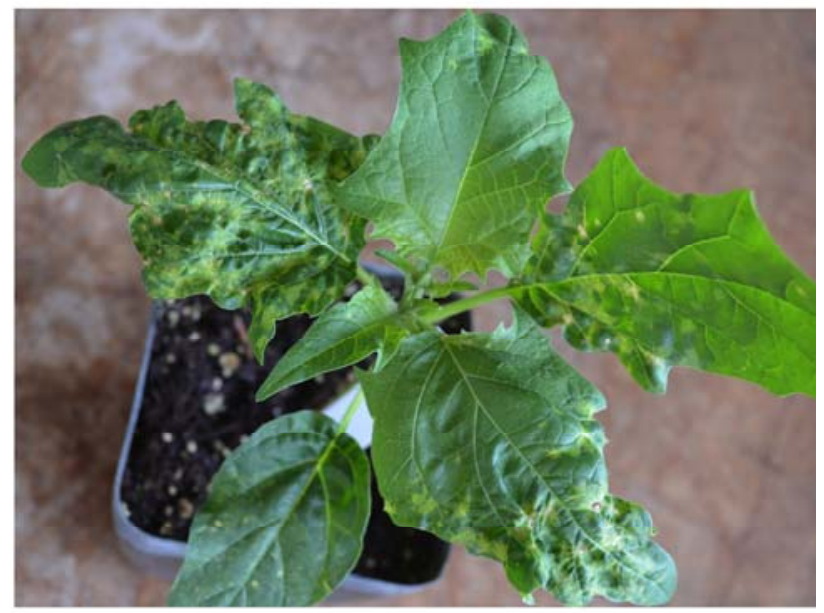

TBSV-Cherry



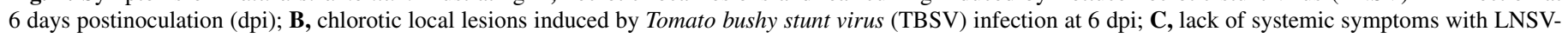
L2 infection at $21 \mathrm{dpi}$; and D, star-shaped lesions resulting from systemic infection by TBSV at $21 \mathrm{dpi}$. 

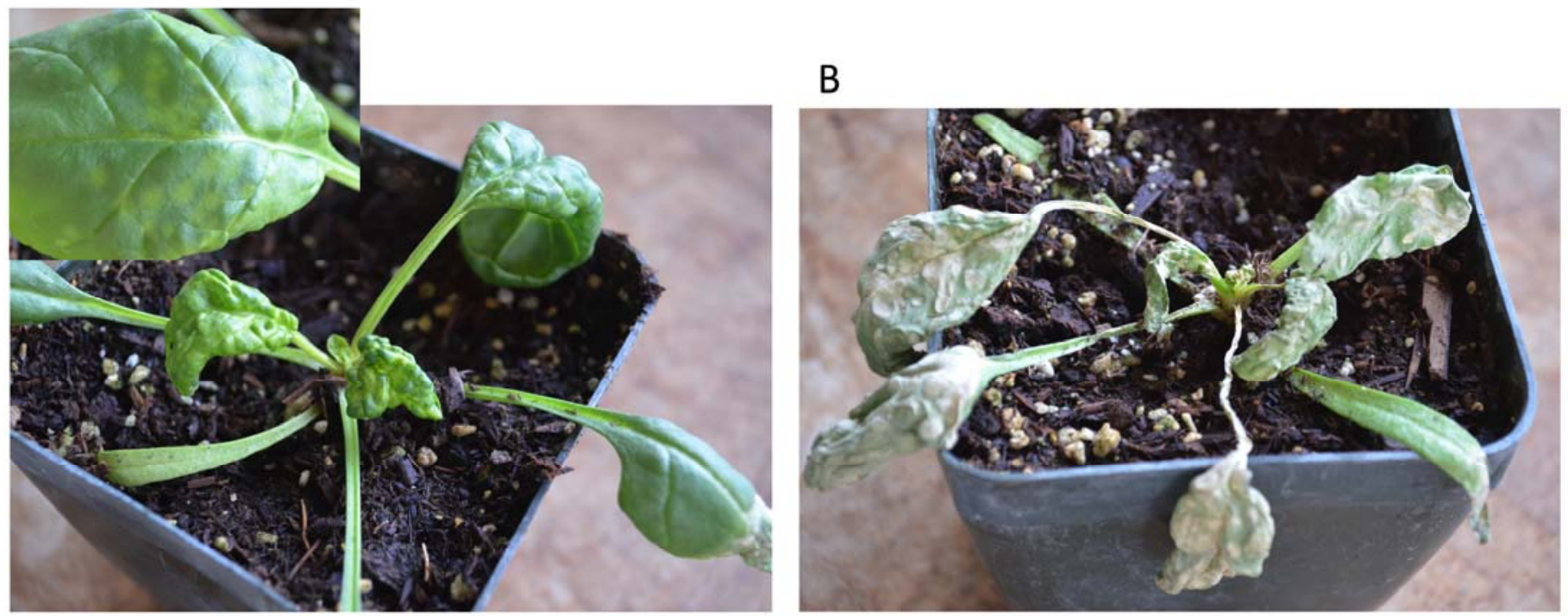

\section{MPV-(LNSV)-L2}

\section{TBSV-Cherry}

Fig. 3. Symptoms on spinach (Spinacea oleracea) at 14 days postinoculation illustrating A, systemic chlorosis and stunting caused by Lettuce necrotic stunt virus (LNSV)-L2 infection and B, plant collapse caused by Tomato bushy stunt virus (TBSV)-Cherry.

(21) indicated a close relationship, and this is supported by near identity throughout the remainder of the LNSV and MPV genomes. Furthermore, host plant reaction to inoculation with LNSVL2, an isolate from California, resulted in host plant reactions in our experiments similar to those uniquely described for MPV upon comparison with TBSV. In particular, infection and symptom development on Chenopodium quinoa and D. stramonium were similar for both LNSV and MPV, and were clearly distinct from results of control inoculations with TBSV.

Interestingly, symptoms of LNSV on Spinacea oleracea differed from observations in previous studies, with plants developing severe stunting, leaf curl, vein clearing, and chlorosis symptoms with MPV infection, whereas TBSV led to necrotic lesions and rapid collapse of plants. Although both viruses ultimately led to plant collapse within 3 weeks, as noted in previous reports $(4,19)$, plants infected with TBSV died within the first 10 days, whereas LNSV-infected plants did not fully collapse until 15 to 20 days. All four replications with LNSV-L2 produced identical symptoms with an absence of necrosis until plants died at $\approx 3$ weeks postinoculation. This could relate to minor differences in the isolate, although this seems unlikely with only $3 \%$ nucleotide sequence variation. More likely, differences relate to either varietal differences among spinach cultivars, environmental conditions during the tests, or perhaps other uncharacterized factors. It should be noted that our experiments were standardized in growth chambers and used inoculum developed from transcript inoculation. This level of standardization was not readily available in the 1970s and early 1980s, during initial characterization of MPV. The advantage of standardization is that it facilitates future comparisons. This is highly important for tombusviruses because symptom severity for these viruses is often greatly influenced by environmental factors. Symptom development for all host plants was highly consistent across all experiments in this study, lending a great deal of confidence for reproducibility under the specified conditions.

Based on the molecular and biological results described herein, we propose that LNSV-L2, the type isolate of LNSV from California, be renamed MPV-L2; that LNSV be recognized as synonymous with MPV; and that MPV be recognized as one of the causative agents of lettuce dieback disease, which causes significant losses to lettuce production in the western United States. Hereafter, LNSV-L2 will be referred to as MPV-L2, and the clone of the L2 isolate will be known as pMPV-L2.

It is interesting that European and African MPV isolates from the 1970s and early 1980s exhibit slightly higher genomic conservation with one another than with the California L2 isolate. Although this variation is slight, it may relate to duration of geographic separation. The two MPV isolates from the RabatSalé region of Morocco and the German isolate were all collected within a period of 6 or 7 years between 1975 and $1982(4,19)$. The MPV-L2 isolate from California was collected in 1997 (14) but the virus may have been in California for several decades. Early lettuce production in California experienced a disease known as brown blight, which closely resembled lettuce dieback and which was prevalent in head lettuce production through the 1930s, severely affecting the heirloom crisphead lettuce varieties 'Iceberg' and 'New York' $(5,8,22)$. No causative agent was ever identified for brown blight $(14,22)$, and herbarium samples have not been identified, but a source of resistance to brown blight was selected from New York and was developed into the resistant lettuce 'Imperial' (9). Imperial replaced New York as the dominant variety in California largely due to disease pressure resulting from brown blight (22). This source of resistance has been maintained in modern crisphead lettuce varieties. Lettuce dieback has never been observed in modern crisphead varieties derived from Imperial (6). A dominant resistance gene preventing tombusvirus infection of lettuce, Tvrl, was identified from 'Salinas' lettuce, a modern crisphead lettuce variety (6), and molecular markers have been developed to facilitate continued selection of resistance $(16,17)$. Both the Tvrl gene and lettuce germplasm derived from Imperial prevent infection by both MPV (LNSV) and TBSV, both of which are known causative agents of lettuce dieback (6). Furthermore, New York lettuce exhibits the same susceptibility to MPV-L2 infection as was described for brown blight (22). This supports our belief that brown blight and lettuce dieback are the same disease, and that MPV, TBSV, or their predecessors have been present in western U.S. lettuce production for at least most of the past century.

Although difficult to confirm, it is possible brown blight may have been caused by the original source of MPV in California. 
A

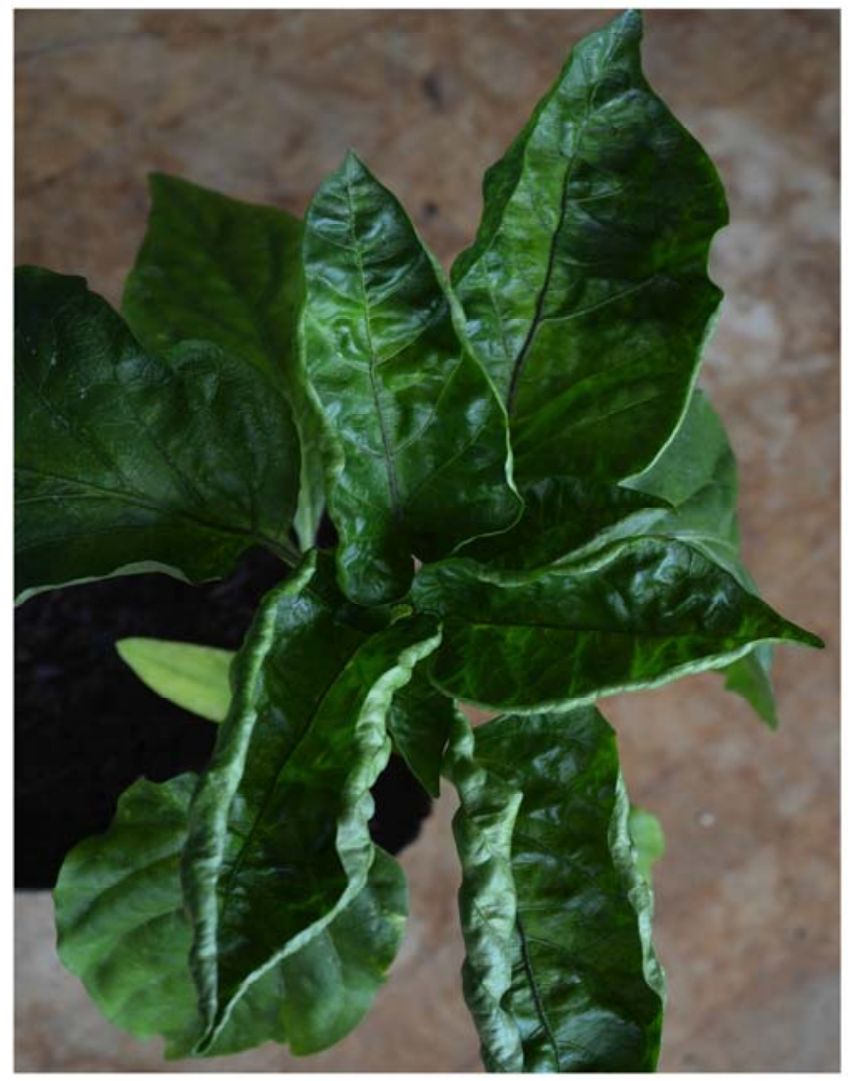

MPV-(LNSV)-L2
B

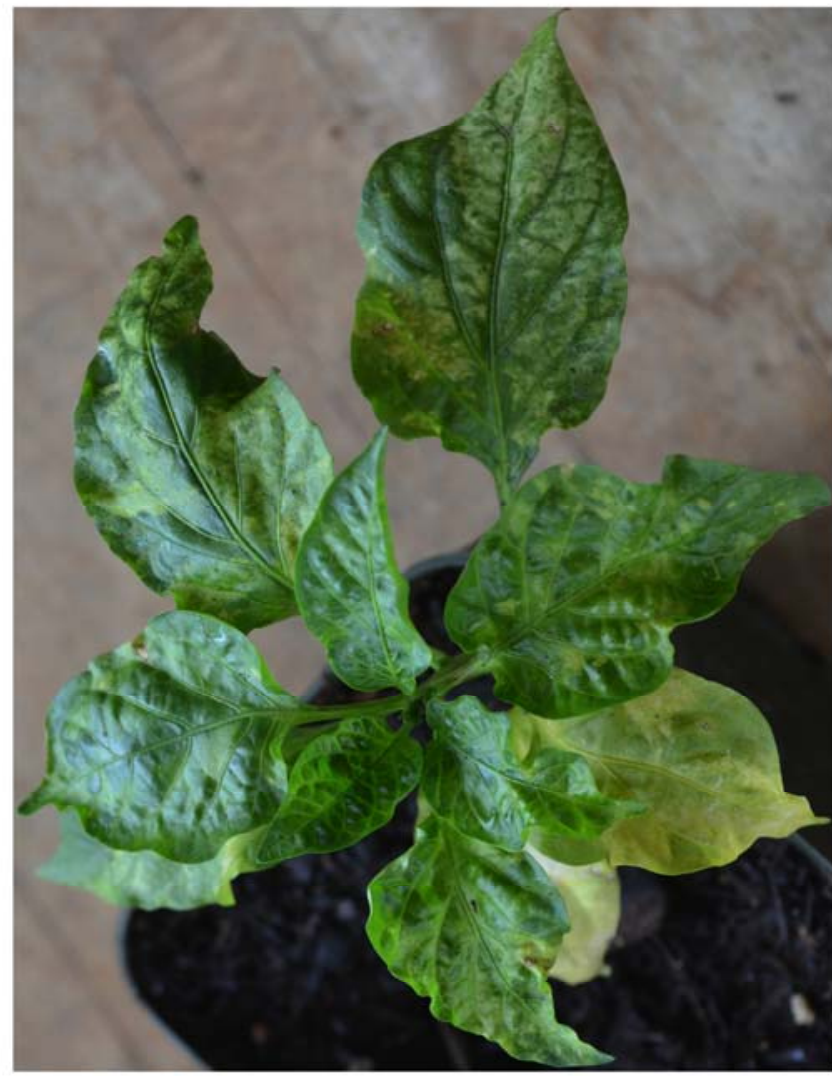

TBSV-Cherry

Fig. 4. Symptoms on bell pepper (Capsicum annuum 'California Wonder') at 28 days post inoculation illustrating A, mosaic, light green chlorosis, and apical leaf curling resulting from Lettuce necrotic stunt virus (LNSV)-L2 infection; and $\mathbf{B}$, mosaic, chlorotic bleaching, and leaf distortion characteristic of infection by Tomato bushy stunt virus (TBSV)-Cherry.

The virus could have originated in either California or Mediterranean regions but genetic isolation and exposure to hosts unique to western North America, Africa, or Europe could have produced the limited genetic variation found between the North American L2 isolate and the isolates from Morocco and Germany. Tombusvirus virions are exceptionally stable, have substantial host ranges, and can maintain infectivity for long periods not only in crop debris but also in water $(10,11,18)$. This stability would facilitate movement on plant material or agricultural equipment both within a region and throughout the world.

The ability of MPV and other tombusviruses to persist as threats to lettuce production in California for decades should raise concern in other parts of the world. MPV was recently identified in Iran $(2,15)$, and appears to be established in soils there just as it is in California. Previous studies isolated viable tombusvirus particles from rivers and lakes $(11,18)$, and limited studies in our laboratory demonstrated that purified L2 virions can remain infectious after storage in sterile, unbuffered water for at least 2 months at $25^{\circ} \mathrm{C}$ (W. M. Wintermantel, unpublished data). The ability of MPV to survive harsh conditions existing in soil and water once introduced raises concern that this virus should be monitored more closely. Use of virus-specific or degenerate RTPCR detection would be a suitable approach for identification of MPV and, potentially, other soil- and water-borne tombusviruses, because significant variability within the coat protein among Tombusvirus spp. leads to challenges for serological detection of tombusviruses distantly related to the source of antiserum used in testing. The ability of tombusviruses to induce symptoms on host plants is often environmentally influenced, and disease develop- ment in susceptible crops may not occur each season, reducing potential opportunities for virus identification. Because MPV has been identified in field agriculture on four continents, and likely exists in other parts of the world, monitoring of affected crops such as lettuce, pepper, spinach, and other hosts would be advisable.

\section{ACKNOWLEDGMENTS}

New sequence accession numbers: JX182425, JX197070, and JX197071. We thank H. J. Vetten, R. Koenig, and M. Russo for providing the MPV isolates; H. B. Scholthof for providing the infectious clone of TBSV-Cherry; and H. J. Vetten and H. B. Scholthof for helpful scientific discussion.

\section{LITERATURE CITED}

1. Beier, H., and Grimm, M. 2001. Misreading of termination codons in eukaryotes by natural nonsense suppressor tRNAs. Nucleic Acids. Res. 29:4767-4782.

2. Beikzadeh, N., Peters, D., and Hassani-Mehraban, A. 2011. First report of Moroccan pepper virus (MPV) on lisianthus in Iran and worldwide. Plant Dis. 95:1485.

3. Dellaporta, S., Wood, J., and Hicks, J. B. 1983. A plant DNA minipreparation: Version II. Plant Mol. Biol. Rep. 1:19-21.

4. Fischer, H. U., and Lockhart, B. E. L. 1977. Identification and comparison of two isolates of tomato bushy stunt virus from pepper and tomato in Morocco. Phytopathology 67:1352-1355.

5. Grube, R. C., Ryder, E. J., Koike, S. T., McCreight, J. D., and Wintermantel, W. M. 2003. Breeding for resistance to new and emerging lettuce diseases in California. Pages 37-42 in: Eucarpia Leafy Vegetables 2003. T. J. L. van Hintum, A. Lebeda, D. Pink, and J. W. Schut, eds. 
Eucarpia, Noordwijkerhout, The Netherlands.

6. Grube, R. C., Wintermantel, W. M., Hand, P., Aburomia, R., Pink, D. A. C., and Ryder, E. J. 2005. Genetic analysis and mapping of resistance to lettuce dieback: A soilborne disease caused by tombusviruses. Theor. Appl. Genet. 110:259-268.

7. Hearne, P. Q., Knorr, D. A., Hillman, B. I., and Morris, T. J. 1990. The complete genome structure and synthesis of infectious RNA from clones of Tomato bushy stunt virus. Virology 177:141-151.

8. Jagger, I. C. 1940. Brown blight of lettuce. Phytopathology 30:53-64.

9. Jagger I. C., Whitaker, T. W., Uselman, J. J., and Owen, W. M. 1941. The Imperial strains of lettuce. U. S. Dep. Agric. Circ. 596.

10. Koenig, R. 1988. Detection in surface waters of plant viruses with known and unknown natural hosts. Pages 305-313 in: Developments in Applied Biology. II. Viruses with Fungal Vectors. J. I. Cooper and M. J. C. Asher, eds. J. I. Association of Applied Biologists, Wellesbourne, UK.

11. Koenig, R., and Lesemann, D.-E. 1985. Plant viruses in German rivers and lakes. I. Tombusviruses, a potexvirus and carnation mottle virus. Phytopathol. Z. 112:105-116.

12. Liu, H.-Y., Sears, J. L., Obermeier, C., Wisler, G. C., Ryder, E. J., Duffus, J. E., and Koike, S. T. 1999. First report of tomato bushy stunt virus isolated from lettuce. Plant Dis. 83:301.

13. Makkouk, K. M., Koenig, R., and Lesemann, D.-E. 1981. Characterization of a tombusvirus isolated from eggplant. Phytopathology 71:572-577.

14. Obermeier, C., Sears, J. L., Liu, H.-Y., Schlueter, K. O., Ryder, E. J., Duffus, J. E., Koike, S. T., and Wisler, G. C. 2001. Characterization of distinct tombusviruses that cause diseases of lettuce and tomato in the western United States. Phytopathology 91:797-806.
15. Rasoulpour, R., and Izadpanah, K. 2011. Isolation and partial characterization of Pelargonium leaf curl virus, Moroccan pepper virus and Eggplant mottled crinkle virus from plant and soil in Iran. J. Phytopathol. 159:802-804.

16. Simko, I., Pechenick, D. A., McHale, L. K., Truco, M. J., Ochoa, O. E., Michelmore, R. W., and Scheffler, B. E. 2009. Association mapping and marker-assisted selection of the lettuce dieback resistance gene Tvr1. BMC Plant Biol. Online publication. doi:10.1186/1471-2229-9-135

17. Simko, I., Pechenick, D. A., McHale, L. K., Truco, M. J., Ochoa, O. E., Michelmore, R. W., and Scheffler, B. E. 2010. Development of molecular markers for marker-assisted selection of dieback disease resistance in lettuce (Lactuca sativa). In: Proc. Int. Soc. Mol. Markers Hortic. N. V. Bassil and R. Martin, eds. Acta Hortic. 859:401-408.

18. Tomlinson, J. A., and Faithfull, E. M. 1984. Studies on the occurrence of tomato bushy stunt virus in English rivers. Ann. Appl. Biol. 104:485-495.

19. Vetten, H. J., and Koenig, R. 1983. Natural infection of tomato and pelargonium in Germany by a tombusvirus originally described from pepper in Morocco. Phytopathol. Z. 108:215-220.

20. White, K. A., and Nagy, P. D. 2004. Advances in the molecular biology of tombusviruses: Gene expression, genome replication, and recombination. Progress in Nucleic Acid Res. Mol. Biol. 78:187-134.

21. Wintermantel, W. M., and Anchieta, A. G. 2012. The genome sequence of Lettuce necrotic stunt virus indicates a close relationship with Moroccan pepper virus. Arch. Virol. Online publication. doi:10.1007/s00705-0121307-x

22. Wisler, G. C., and Duffus, J. E. 2000. A century of plant virus management in the Salinas Valley of California, 'East of Eden.' Virus Res. 71:161-169. 